\title{
Molecular and Clinical Characteristics Associated with Elevated TREM1 and its Emergence as a Prognostic Biomarker in Gliomas
}

\section{Lin Zhang}

Shandong University

Yangyang Xu ( $\nabla$ drxuyang@sdu.edu.cn )

Shandong University https://orcid.org/0000-0003-1862-7090

Xun Qu

Shandong University

\section{Primary research}

Keywords: Glioma, TREM1, Prognosis, Immune response, Tumor microenvironment

Posted Date: October 12th, 2021

DOI: https://doi.org/10.21203/rs.3.rs-958851/v1

License: (c) (i) This work is licensed under a Creative Commons Attribution 4.0 International License.

Read Full License 


\section{Abstract}

\section{Background:}

Monotherapy with immune checkpoint blockade for most gliomas does not seem to induce durable antitumor responses, which drive us to explore potential immunological targets in glioma. Triggering receptor expressed on myeloid cells-1 (TREM1) has been reported to be associated with immune-related inflammatory disorders. However, to date, there has rarely been studied in glioma.

\section{Methods:}

The data included clinicopathological characteristics and transcriptome data of patients with glioma obtained from publicly available genomic databases. Bioinformatics methods were employed to identify TREM1 expression pattern, prognostic value, biological function, and immune characteristics by $R$ language, SPSS 19 and GraphPad Prism 8 software

\section{Results:}

In this study, it was observed that TERM1 expression was increased significantly in GBM compared with other types and was associated with poor outcome. Increased TREM1 accompanied distinct mutation and amplification of driver oncogenes. Moreover, gene ontology and KEGG analyses showed that TREM1 may play a role in the immunobiology of gliomas. TREM1 may participate in the immunosuppressive microenvironment by inhibiting $T$ cell antitumor immunity functions. Additionally, through correlation analysis, TREM1 was found to be tightly correlated with immune checkpoint molecules.

\section{Conclusion:}

TREM1 may be a novel and attractive immune target and may provide new avenues to overcome the low response rate of glioma patients to current immunotherapies.

\section{Background}

Glioma is the most incidence and deadliest type of primary malignant tumor in the central nervous system (CNS)[1]. Although it has made great advances in multimodal conventional therapy, including total resection plus adjuvant radio- chemotherapy, the five-year survival rate is still less than $10 \%[2]$. Glioma patients succumb in large part due to the rapid growth of tumors, high invasiveness, and resistance to treatments[3]. Recently, with the deep-going research, clinical trials of immunotherapies that target the immune components of tumor microenvironments have shown exceptional promise for the treatment of cancer[4]. Meanwhile, the lymphatic vasculature is discovered in the CNS, which has provided a new theoretical basis and sheds light on glioma immunotherapy[5].

A large number of studies have demonstrated that a breakthrough in cancer immunotherapy is emerging with drugs that target immune checkpoint molecules. Under physiological circumstances, immune 
checkpoints play a crucial role in maintaining self-tolerance and in tuning the duration and amplitude of immune responses. Through the breakdown of this delicate balance, cancer is able to counteract immune system attacks by usurping the mechanisms that usually prevent autoimmunity. Immune checkpoint inhibitors (ICls) and monoclonal antibodies (mAbs) targeting inhibitory receptors have been subjected to clinical trials, and striking success has been achieved across multiple solid tumors[6-8]. However, monotherapy with immune checkpoint blockade for most gliomas does not seem to induce durable antitumor responses [9]. It has been demonstrated that ICl-combined drug therapy can exhibit remarkable advantages and efficacy. In pancreatic ductal adenocarcinoma, IL17 blockade increased immune checkpoint blockade (PD-1, CTLA4) sensitivity[10]. In breast and colon cancer-bearing mouse models, CCR4 inhibition enhanced the antitumor effect of anti-CTLA-4 and resulted in long-lasting immunity[11]. These exciting results inspired us to explore other potential immunological targets in the glioma microenvironment.

The TREM receptor family, which belongs to the Ig-like superfamily, has been demonstrated to be involved in inflammatory diseases, autoimmune disorders, and cancers [12]. Among them, triggering receptor expressed on myeloid cells-1 (TREM1) is the first identified and best-characterized family member. TREM1 plays roles in the release of proinflammatory cytokines, T-cell differentiation, and effector responses[13]. Studies have shown that TREM1 is associated with the occurrence of immunerelated inflammatory diseases, such as arthritides, pancreatitis, and bowel inflammatory disorders[14, 15]. TREM1 mRNA and protein upregulation was accompanied by an increased inflammatory response and disease severity in mouse models of colitis, which were attenuated in TREM1-deficient mice[16]. Recent investigations have highlighted the important influence of TREM1 signaling on inflammationmediated carcinogenesis. High TREM1 expression levels in lung adenocarcinoma and hepatocellular carcinoma were suggested to be independent predictors of tumor progression and poor patient prognosis $[17,18]$. It could be possible to diagnose early cancers in a broad population by assessing sTREM concentrations levels in biological fluids[19]. A TREM1 inhibitor could sensitize tumors to immunotherapy in a model of hepatocellular carcinoma by synergistically reversing immunosuppression and reactivating effector T cells to exert tumor cell cytotoxicity [20]. Therefore, TREM1 may be a novel and attractive immune target and may exhibit new avenues to overcome the low response rate of glioma patients to current immunotherapies.

To date, there is no comprehensive report illustrating the immunosuppressive status with different TREM1 expression levels in glioma. In this sense, an in-depth exploration of the immunobiological processes of TREM1 based on the current genomic databases can lead to a better understanding of the complexity of the tumour immune microenvironment. Here, we investigated the clinical, molecular, and immunological characteristics of TREM1 in glioma. Our results demonstrated that TREM1 was highly expressed in GBM and was a valuable prognostic biomarker for glioma patients. Moreover, TREM1 participated in the immunosuppressive response, correlated with tumor-infiltrating immune cells and synergistic with several immunosuppressive members. In our study supports TREM1 was identified as a potential immune molecular target to further optimize immunotherapy for glioma. 


\section{Materials And Methods \\ Data retrieval and preprocessing}

The data included clinicopathological characteristics and transcriptome data of patients with glioma (WHO grades II-IV) obtained from the Cancer Genome Atlas (TCGA) database (RNA-seq, $n=669$ ), the Chinese Glioma Genome Atlas (CGGA) database (RNA-seq, $n=693$, microarray, $n=301$ ), Gene Expression Omnibus database (GSE16011, $n=268$ ), the Gill database $(n=92)$, IVY Glioblastoma Atlas Project data $(n=270)$, and the Rembrandt database $(n=580)$. Somatic copy number alternations (CNAs) and somatic mutations were downloaded from the TCGA data portal. GISTIC 2.0 was employed to analyze relationship between CNAs and TREM1 expression. The TREM1 protein expression level was obtained online from the Human Protein Atlas.

\section{Bioinformatic analysis}

All transcriptome data were log2 transformed. Genes significantly related to TREM1 expression were analyzed by Pearson correlation analysis. Gene ontology analysis was performed when related gene sets were submitted to the DAVID website (http://david.abcc.ncifcrf.gov/home.jsp). Gene set enrichment analysis (GSEA) (https://www.broadinstitute.org/gsea/index.jsp) was used to identify differential phenotypes between the low- and high-TREM1 groups. Stromal and immune scores and tumor purity of glioma tissue were calculated using the ESTIMATE R package. T cell specific genesets were downloaded from the AmiG02 Web portal (http://amigo.geneontology.org). Principal component analysis (PCA) and gene set variation analysis (GSVA) were used to analyze profile patterns of the transcriptome, immune function and inflammation in high or low TREM1 expression cases.

\section{Statistical analysis}

Differences in variables between the two groups were assessed using the Student's t-test, or a one-way analysis of variance (One-way ANOVA) with at least three groups. Kaplan-Meier survival curves were used to chart survival distributions and statistical significance between two groups was assessed by log-rank tests. Univariate and multivariate Cox regression analyses were performed to identify independent prognostic factors. R package files were used to produce the figures, including ggplot2, corrgram, circlize, and nomogram. SPSS 19 and GraphPad Prism 8 software were employed for statistical analysis. $\mathrm{P}<$ 0.05 was considered statistically significant.

\section{Results}

\section{Associations of TREM1 expression with molecular characteristics in gliomas}


Expression data from publicly available databases (TCGA RNA-seq, $n=669$; CGGA RNA-seq, $n=693$; CGGA microarray, $n=301$; and GSE16011, $n=268$ ) were used to evaluate the expression levels of TREM1 mRNA in gliomas of different WHO grades. TERM1 was observed to be increased significantly in GBM compared to grade II and grade III glioma samples (Fig. 1A). In the TCGA RNA-seq and CGGA mRNA microarray datasets, the TREM1 mRNA expression level was also higher in WHO grade III samples than in WHO grade II samples (Fig. 1A). Moreover, TREM1 expression was elevated with histopathologic grades (Supplementary Fig. S1). Gliomas are classified into five types in the 2016 WHO CNS tumor classification: LGG-Oligo (lower-grade glioma oligodendroglioma with IDH mutation and TERT promoter mutation or 1p/19q codeletion); LGG-Astro (LGG astrocytoma with IDH mutation without TERT promoter mutation or $1 p / 19 q$ codeletion, and with ATRX mutation); LGG IDHwt (LGG with wild-type IDH status); GBM IDHmut (GBM with mutant IDH status); and GBM IDHwt (GBM with wild-type IDH status)[1]. LGGOligo had the lowest TREM1 expression, while GBM IDHwt showed the highest expression (Fig. 1B). The TREM1 expression level in glioma cases with a wild-type IDH status was detected higher than those in cases with mutant IDH in both TCGA and CGGA datasets (Supplementary Fig. S2). Additionally, we found that TREM1 protein was the most strongly overexpressed protein in HGG compared with LGG and normal brain tissues from the Human Protein Atlas (Fig. 1C). These findings indicated that higher TREM1 expression was accompanied by higher malignancy in glioma.

Integrated genomic- and genetic-based classification offers a new perspective for predicting outcomes in patients with different gliomas[21]. We then investigated TREM1 expression levels in TCGA four molecular subtypes. As shown in Fig. 1D, compared to the other three subtypes, TREM1 was upregulated dramatically in the mesenchymal subtype in the Rembrandt dataset, as well as in the CGGA dataset. The discrimination ability of TREM1 expression for mesenchymal subtype in all grade gliomas was further assessed by Receiver operating characteristic curve (ROC) analysis. The area under the curve (AUC) of TREM1 expression was 0.8906 in the Rembrandt cohort. A similar result was also observed in the CGGA cohort (AUC 0.9887, P< 0.001), while it was 0.6312 in the TCGA cohort (Supplementary Fig. S3). These results suggested that TREM1 may serve as a biomarker for mesenchymal subtype in gliomas. Then, we analyzed the intratumor distribution of TREM1 in GBM tissues. MRI-localized biopsies revealed that GBM tissues from the contrast-enhancing (CE) core of tumors had different cellular and molecular compositions compared with tissues from the non-enhancing (NE) margins of tumors[22]. We observed that GBM-CE regions had higher TREM1 expression than NE or nonneoplastic areas by analyzing 93 GBM samples RNA sequencing data [22] (Fig. 1E). Furthermore, based on IVY Glioblastoma Atlas Project data $(n=270)$, TREM1 was found to be enriched in pathological areas that were important for glioma progression compared to other areas, including HBV (hyperplastic blood vessels), PAN (pseudopalisading cells around necrosis) and PNZ (perinecrotic zone) (Fig. 1F).

\section{TREM1 predicts worse survival in glioma patients}

Kaplan-Meier analysis was further performed to investigate the prognostic value of TREM1. As shown in Fig. 2A and 2B, patients with high TREM1 expression glioma exhibited significantly shorter overall 
survival (OS) than their counterparts in pan-glioma analysis of TCGA and CGGA datasets. In addition, similar results were validated in the two cohorts of patients with WHO grade III and IV gliomas, although the grade II subgroup failed to reach statistical significance. Furthermore, univariate and multivariate Cox regression analyses were employed to validate TREM1 as an independent prognostic marker in gliomas. Univariate Cox analysis showed that high TREM1 expression, grade, age, IDH mutation, and chemotherapy were significantly associated with overall survival in the CGGA database. Multivariate Cox regression analysis revealed that TREM1 expression was still an independent predictor for glioma patients (HR: 1.046; 95\% Cl: 1.019-1.117; $\mathrm{P}=0.045)$ after adjusting for the clinical factors mentioned above (Table S1). According to the univariate Cox regression, this prognostic value only significant in the TCGA dataset (Table S2). A nomogram, based on statistical calculation of the risk of clinicopathological features of a cancer, has been proven to be accurate with visualization and quantification for doctors and patients, and has been widely developed to predict patient survival in the clinic[23]. In this study, a nomogram model was further constructed to predict 1-year, 3-year, and 5-year survival based on the TCGA cohort (Fig. 2C). Meanwhile, the calibration curves showed that the actual observation fit well with the predicted glioma patients' survival probability (Fig. 2D). All these findings collectively implied that TREM1 may serve as an independent prognostic biomarker for glioma patients.

\section{TREM1 expression correlates with distinct genomic changes}

To explore the molecular characteristics associated with the expression pattern of TREM1, copy number alterations and somatic mutations from TCGA database were collected. First, somatic copy number alterations were evaluated between the high and low TREM1 expression groups. As shown in Fig. 3A and $3 B$, the incidence of $1 p / 19 q$ codeletion was reduced with increasing TREM1 expression, which is a genomic characteristic of oligodendroglioma[24]. Chr7 amplification accompanied by Chr10 deletion, a frequent genomic event in GBM[25], was enriched in the high TREM1 expression group. We analyzed all glioma cases using GISTIC 2.0 to identify 42 significantly reoccurring focal amplifications and 54 deletion events (Supplementary Dataset S1). In the high TREM1 expression group, focal amplification peaks, including PIK3C2B (1q32.1), PDGFRA (4q12), EGFR (7p11.2), and CDK4 (12q14.1), were wellcharacterized oncogenic driver genes, while this group was accompanied by a focal deletion peak in 9p21.3 (CDKN2A and CDKN2B). Additionally, somatic mutations were also analyzed based on TREM1 expression. A higher frequency of mutations in IDH1 (40\%), TP53 (39\%), ATRX (22\%), and PTEN (19\%) was observed in the high TREM1 expression cases (Fig. 3C). As shown in Fig. 3D, the forest plot further revealed that mutations in RYR2, SVEP1, L1CAM, PTEN, SMARCA4, PCDH19, CHL1, and CFAP47 were significantly enriched in the high TREM1 expression cases, while the low group frequently mutated in IDH1 and ATRX.

\section{TREM1-related biological functions in glioma}


To reveal the biological functions of glioma with different TREM1 expression levels, the genes that were strongly correlated with TREM1 expression (Pearson $r>|0.5|, P<0.05$ ) were selected in the TCGA and CGGA databases (Supplementary Dataset S2). Then, the related genes were explored by $\mathrm{GO}$ analysis in DAVID Bioinformatics Resources 6.8[26]. GO analysis results obtained with the genes positively associated with TREM1 expression from the TCGA dataset revealed that these functions were mostly involved in inflammation and immunity, such as inflammatory response, immune response, leukocyte migration, and chemotaxis (Fig. 4A). The CGGA database yielded similar results as well (Fig. 4B). As mentioned above, the highest TERM1 expression level was in GBM, and we employed GBM cases to perform further investigation. Supplementary Figure S4 illustrated that those related genes still played a role in immune responses and inflammation. Then, GSEA was employed to validate the biological function of TREM1, showing that the cases with high TREM1 expression had an activated phenotype for angiogenesis processes and inflammatory response in the TCGA and CGGA datasets (Fig. 4C and D). PCA also showed that angiogenesis processes and inflammatory responses were generally different based on TREM1 expression status (Fig. 4E and 4F). In addition, based on KEGG pathology data, the majority of the positive genes associated with TREM1 expression were involved in immune-related pathways (Supplement Fig. S5). The signaling network according to the results from KEGG pathway analysis suggested an association between TREM1 and inflammatory-immune related pathways, such as NF-KB signaling, TNF signaling, Toll-like receptor signaling, and chemokine signaling pathways (Fig. 5A and Supplement Fig. S6).

To further explore TREM1 involvement in the immune response in glioma, we downloaded the immune response genesets from the AmiGO2 Web portal (http://amigo.geneontology.org/). Collectively, heatmaps was drawn using the genes correlated with TREM1 expression (Pearson $r>|0.4|, P<0.05$ ) in the TCGA and CGGA databases (Fig. 5B and 5C). A total of 708 genes in the TCGA database showed positive correlation with TREM1, while 98 genes displayed a negative correlation. In the CGGA dataset, the number of positively related genes was 613 , and the counterpart was just 11 . Detailed information about these genes was provided in Supplementary Dataset S3. The above analysis implied that TREM1 was positively correlated with most immune responses in glioma. Taking these findings together, TREM1 may play an important role in immunobiologic processes of gliomas.

\section{Association of TREM1 expression with tumor purity and immune and stromal cell populations in the glioma microenvironment}

Just like other cancers, glioma tissues also are composed of not only neoplastic cells but also nonneoplastic cells, such as stromal cells and immune cells. It was reported that these nonneoplastic cells diluted the purity of glioma and played important roles in the progression of gliomas [27]. For a thorough understanding of the relationship between neoplastic and non-neoplastic cells, the ESTIMATE algorithm method was designed[28]. We found that TREM1 expression was negatively correlated with 
tumor purity (TCGA-seq: $\mathrm{R}=-0.7350, \mathrm{P}<0.0001$; CGGA-seq: $\mathrm{R}=-0.7126, \mathrm{P}<0.0001$ ) in the TCGA and CGGA datasets (Fig. 6A and 6B). Similar results were also validated in GBM cases from TCGA and CGGA databases (Supplement Fig. S7A and S7B). To further evaluate the relationship between TREM1 and nontumor cells in the glioma microenvironment, the relationship between TREM1 and 64 immune and stromal cell types was determined using the xCell method[29]. As shown in Fig. 6C and 6D and Supplementary Dataset S4, TREM1 positively correlated with multiple infiltrating immune cell types, such as monocytes, macrophages, DCs, and CD4+ memory T-cells. In contrast, TREM1 was negatively associated with Tregs and CD4+ Tcm. In addition, stromal cell types were also enriched in high TREM1 glioma cases, including epithelial cells and mesangial cells. Furthermore, GBM cases from TCGA and CGGA datasets also showed similar results (Supplement Fig. S7C and S7D, and Supplementary Dataset S4). Taken together, these data suggested that TREM1 may participate in the regulation of immune and stromal cells in the glioma microenvironment.

\section{TREM1-related T cell immunity and inflammatory activities in glioma}

Circumstantial evidence indicates that TREM1 plays important roles in the amplification of the immuneinflammatory response via cytokine production and the regulation of antigen-presenting cells and T-cell activation[30]. Accordingly, we employed GSVA analysis to evaluate whether TREM1 might be involved in $T$ cell immunity in gliomas. We found that TREM1 was positively correlated with positive regulation of $T$ cell tolerance induction (G0:0002666), positive regulation of T cell cytokine production (GO:0002726), positive regulation of regulatory $T$ cell differentiation (G0:0045591), and positive regulation of $T$ cell activation via $\mathrm{T}$ cell receptor contact with antigen bound to $\mathrm{MHC}$ molecule on antigen presenting cell (GO:2001190) in both of the TCGA and CGGA cohorts. Moreover, TREM1 was negatively correlated with T cell-mediated immune response to tumor cells (GO:0002424), regulation of T cell-mediated immune response to tumor cells (G0:0002840), and positive regulation of T cell-mediated immune response to tumor cells (G0:0002842) in the TCGA cohort (Fig. 7A and 7B, Supplementary Dataset S5). These results implied that TREM1 may play an important role in inhibiting $T$ cell antitumor immunity functions in the glioma microenvironment.

Based on the results mentioned above indicating that TREM1 were involved in the immune response, we explored the role of TREM1 in inflammation using seven metagenes as previously described[31]. In both the TCGA and CGGA datasets, TREM1 expression was found to be positively correlated with HCK, interferon, LCK, MHC-I, MHC-II, and STAT1 metagenes but negatively correlated with IgG metagenes in gliomas (Fig. 7C and 7D). These findings indicated that TREM1 was abundant in macrophage activation, T-cell signaling transduction and antigen-presenting cells, but weakly associated with B lymphocyterelated immune response. 


\section{TREM1 affects glioma-induced immunity in a synergistic manner with other immune checkpoint members}

Immune checkpoints are extremely important molecules in the regulation of immune processes. Therefore, we next examined the correlationship between TREM1 and immune checkpoints expression. We enrolled 29 well-known immune checkpoints in this analysis, including the B7-CD28 family (PD-L1, PD-L2, ICOSLG, B7-H3, B7-H4, HHLA2, CTLA2, ICOS, PD-1, and TGMIGD2), TNF superfamily (BTLA, LIGHT, CD40, OX40, 4-1BB, CD27, CD40LG, 4-1BB-L, CD70, and AITR), and other immune checkpoint members (TIM3, ID01, LAG3, FGL1, CD39, CD73, SIGLEC15, VSIR, and NCR3)[32]. The expression heatmap of these molecules was mapped, taking other clinical features into account, such as IDH and grade (Supplement Fig. S8 and Supplementary Dataset S6). We found that TREM1 expression was positively correlated with most of these immune checkpoint molecules in both the CGGA and TCGA cohorts, implying that TREM1 may be synergistic with other checkpoint molecules to regulate the immune response in gliomas. Furthermore, TREM1 was assessed in relation to several well-known immune checkpoint genes in gliomas. TREM1 was highly correlated with TIM-3, CTLA4, LAG3, VISTA, ID01, BTLA, B7-H3, and PD-1 in glioma, LGG and GBM cases alone in the TCGA datasets. Similar results were also verified in the CGGA database (Fig. 8). In light of the findings, it was speculated that combined treatment with TREM1 and immune checkpoints may be effective in overcoming the limitations of using immune checkpoints alone.

\section{Discussion}

The management of advanced-stage malignancies has been greatly transformed by the development of cancer immunotherapy. Immune checkpoint blockade therapy has revolutionized the treatment of several tumor subtypes[33]. However, the antitumor effect of monotherapy with immune checkpoint inhibitory agents is limited to certain types of cancer, such as gliomas, with an effective response rate of less than $30 \%[34,35]$. It is these unfavorable results that encourage us to identify other immune targets and optimize treatments strategies.

TREM1 has recently been identified as a new potential target for immunotherapy. It has been demonstrated that TREM1 inhibitors can attenuate tumor growth and promote the antitumor efficacy of blocking PD-L1[20]. However, to date, there has been no systematic research on TREM1 in glioma. In this study, a large-scale, comprehensive, bioinformatic analysis was used to characterize the landscape of TREM1 among gliomas. We observed that TREM1 expression increased with the grade of gliomas. TREM1 was enriched in the mesenchymal subtype and significantly upregulated in IDH wild-type gliomas. TREM1 was localized to perinecrotic regions, pseudopalisading cells around necrosis, and hyperplastic blood vessels. In addition, our study suggested higher TREM1 expression levels predicted worse survival among patients with glioma, especially GBM. Importantly, TREM1 was a valuable independent prognostic factor in glioma. There appears to be an association between TREM 1 and a malignant phenotype and that it may be involved in glioma progression. 
We then conducted GO and KEGG analyses to understand the main biological processes and pathways associated with TREM1 in whole gliomas. We found that TREM1 was closely associated with inflammatory activities and immune responses, similar to PD-L1, B7-H3, and CTLA4. Our analysis also showed that TREM1 was a critical player in regulating immune-related pathways, such as NF-KB and TNF signaling, which were essential for controlling the transcription of key genes. Our results are consistent with what has been reported about TREM1. Recent evidence has demonstrated that both in vivo and in vitro, TREM1 can activate the downstream proinflammatory pathways CARD9/NF-KB and NLRP3/caspase- 1 to facilitate the release of intracellular inflammatory factors and yield poststroke neuroinflammatory damage[36]. TREM1 ablation diminished the activation of the p38, ERK1/2, JNK, MAPK, and NF-KB signaling pathways and downregulated the transcription and protein expression of IL-6, IL-1 $\beta$, TNF, CCL2, and CXCL10, indicating that TREM1 as a key determinant of Kupffer cell activation in hepatocarcinogenesis [37]. Taken together, these investigations suggested that TREM1 is a key regulator of immune and inflammatory responses and an indicator of tumor development.

Studies have revealed the importance of tumor purity in clinical features and identified the resulting enriched immune cells. Low purity indicates gliomagenesis, malignancy progression, and an enhanced immune phenotype[38]. We found that TREM1 was closely associated with immune score, stroma score, and microenvironment score, indicating high TREM1 expression with low purity and complexity of the glioma microenvironment. Consistently, based on the correlation analysis between TREM1 and microenvironment components, high TREM1 was accompanied by high levels of immune infiltration, including monocytes, CD8+ and CD4+ T-cells, DCs, and NK cells. Moreover, TREM1 could promote CD4+ T-cell proliferation and lead to CD8+ T-cell deletion, subsequently increasing the CD4/CD8 T-cell ratio at the site of inflammation[39-41]. TREM1 was shown to recruit Foxp3+ Treg infiltration, mediate CD8+ Tcell dysfunction, and finally facilitate immunosuppression in mice[20]. In addition, it was observed that TREM1 transcript levels were associated with immunosuppressive cells such as macrophages and MDSCs. Biological and functional assays showed that TREM1+ TAMs had higher expression of programmed cell death ligand 1 (PD-L1), which abrogated spontaneous and PD-L1 blockade-mediated antitumor effects in vivo[20]. In summary, these data suggested TREM1 functions as a contributor to the immunosuppressive microenvironment and malignant progression of gliomas.

The preclinical benefit of cancer therapy has been demonstrated by a number of immune checkpoint inhibitors[42], and clinical trials of immune checkpoint blockades for gliomas are ongoing[43, 44]. Following that, we investigated the relationship between TREM1 and immune checkpoint molecules. TREM1 was strongly synergistic with B7-H3, B7-H4, PD-L1, TIM3, and PD-1 in both pan-glioma analysis and different grade glioma specimens. Evidence has revealed that TREM1 inhibitors can sensitize tumor cells to anti-PD-L1 immunotherapy for liver cancer[20]. In addition, one clinical trial has already reported that dual PD-1 and CSF-1R blockade may provide anti-tumor effects which are durable for malignant glioma patients (ClinicalTrials.gov identifier: NCT02526017). Although TREM1 inhibitors are currently unavailable for clinical application, a desirable relationship between TREM1 and other immune checkpoints maps out a rosy blueprint for the combination therapy of glioma. 


\section{Conclusion}

The biological significance of TREM1 was investigated in immune responses and tumor progression using large sample-sized glioma cohorts. TREM1 overexpression may help to explain why immunotherapy does not work against malignant gliomas. Therefore, additional functional experiments with TREM1 and exploration of a possible upregulation mechanism is necessary. These findings indicated TREM1 as a novel immune target that may improve clinical efficacy in glioma treatment.

\section{Abbreviations}

ICls: Immune checkpoint inhibitors; TREM1: triggering receptor expressed on myeloid cells-1; TCGA: The Cancer Genome Atlas; CGGA: Chinese Glioma Genome Atlas; GSEA: Gene set enrichment analysis; PCA: Principal component analysis; GSVA: gene set variation analysis; ROC: Receiver operating characteristic curve; AUC: Area under the ROC curve; PD-L1: programmed cell death ligand 1

\section{Declarations}

\section{Acknowledgments}

The authors thank the Cancer Genome Atlas (TCGA), the Chinese Glioma Cooperative Group (CGCG), IVY Glioblastoma Atlas Project, Rembrandt database, GEO database, and Human Protein Atlas for kindly providing gene glioma profiling data.

\section{Author contributions}

$L Z, Y Y X$ and $X Q$ conceived and designed the study. LZ and YYX performed the data analysis, wrote the paper. All authors read and approved the manuscript.

\section{Funding}

This work was supported by the National Natural Scientific Fund of China (81772879); the Shandong Provincial Natural Science Foundation (ZR2019PH042).

\section{Data availability statement}

The data supporting the findings in this study can be obtained from the Cancer Genome Atlas (TCGA) database (http://cancergenome.nih.gov/), the Chinese Glioma Genome Atlas (CGGA) database (http://www.cgga.org.cn), IVY Glioblastoma Atlas Project data (http://glioblastoma.alleninstitute.org/) 
,the Rembrandt database (https://caintegrator.nci.nih.gov/rembrandt/), and Human Protein Atlas (www.proteinatlas.org). All processed data and R codes used in this study can be obtained from the corresponding authors on reasonable request.

\section{Ethics approval and consent to participate}

This article does not contain any studies with human participants performed by any of the authors.

\section{Conflict of interest}

The authors confirm that there is no conflict of interests.

\section{Informed consent}

All authors consent to publish the article.

\section{References}

1. Louis DN, Perry A, Reifenberger G, von Deimling A, Figarella-Branger D, Cavenee WK, Ohgaki $H$, Wiestler OD, Kleihues P, Ellison DW. The 2016 World Health Organization Classification of Tumors of the Central Nervous System: a summary. Acta Neuropathol. 2016;131(6):803-20.

2. Tan AC, Ashley DM, Lopez GY, Malinzak M, Friedman HS, Khasraw M. Management of glioblastoma: State of the art and future directions. CA Cancer J Clin. 2020;70(4):299-312.

3. Furnari FB, Fenton T, Bachoo RM, Mukasa A, Stommel JM, Stegh A, Hahn WC, Ligon KL, Louis DN, Brennan C, et al. Malignant astrocytic glioma: genetics, biology, and paths to treatment. Genes Dev. 2007;21(21):2683-710.

4. Qi Y, Liu B, Sun Q, Xiong X, Chen Q. Immune Checkpoint Targeted Therapy in Glioma: Status and Hopes. Front Immunol. 2020;11:578877.

5. Louveau A, Smirnov I, Keyes TJ, Eccles JD, Rouhani SJ, Peske JD, Derecki NC, Castle D, Mandell JW, Lee KS, et al. Structural and functional features of central nervous system lymphatic vessels. Nature. 2015;523(7560):337-41.

6. Weber JS, D'Angelo SP, Minor D, Hodi FS, Gutzmer R, Neyns B, Hoeller C, Khushalani NI, Miller WH Jr, Lao $C D$, et al. Nivolumab versus chemotherapy in patients with advanced melanoma who progressed after anti-CTLA-4 treatment (CheckMate 037): a randomised, controlled, open-label, phase 3 trial. Lancet Oncol. 2015;16(4):375-84.

7. Hodi FS, O'Day SJ, McDermott DF, Weber RW, Sosman JA, Haanen JB, Gonzalez R, Robert C, Schadendorf D, Hassel JC, et al. Improved survival with ipilimumab in patients with metastatic 
melanoma. N Engl J Med. 2010;363(8):711-23.

8. Hamid O, Robert C, Daud A, Hodi FS, Hwu WJ, Kefford R, Wolchok JD, Hersey P, Joseph RW, Weber JS, et al. Safety and tumor responses with lambrolizumab (anti-PD-1) in melanoma. N Engl J Med. 2013;369(2):134-44.

9. Jackson CM, Choi J, Lim M. Mechanisms of immunotherapy resistance: lessons from glioblastoma. Nat Immunol. 2019;20(9):1100-9.

10. Zhang Y, Chandra V, Riquelme Sanchez E, Dutta P, Quesada PR, Rakoski A, Zoltan M, Arora N, Baydogan S, Horne W, et al: Interleukin-17-induced neutrophil extracellular traps mediate resistance to checkpoint blockade in pancreatic cancer. J Exp Med 2020, 217(12).

11. Marshall LA, Marubayashi S, Jorapur A, Jacobson S, Zibinsky M, Robles O, Hu DX, Jackson JJ, Pookot D, Sanchez J, et al: Tumors establish resistance to immunotherapy by regulating Treg recruitment via CCR4. J Immunother Cancer 2020, 8(2).

12. Klesney-Tait J, Turnbull IR, Colonna M. The TREM receptor family and signal integration. Nat Immunol. 2006;7(12):1266-73.

13. Arts RJ, Joosten LA, van der Meer JW, Netea MG. TREM-1: intracellular signaling pathways and interaction with pattern recognition receptors. J Leukoc Biol. 2013;93(2):209-15.

14. Bosco MC, Raggi F, Varesio L. Therapeutic Potential of Targeting TREM-1 in Inflammatory Diseases and Cancer. Curr Pharm Des. 2016;22(41):6209-33.

15. Pelham CJ, Pandya AN, Agrawal DK. Triggering receptor expressed on myeloid cells receptor family modulators: a patent review. Expert Opin Ther Pat. 2014;24(12):1383-95.

16. Weber B, Schuster S, Zysset D, Rihs S, Dickgreber N, Schurch C, Riether C, Siegrist M, Schneider C, Pawelski $\mathrm{H}$, et al. TREM-1 deficiency can attenuate disease severity without affecting pathogen clearance. PLoS Pathog. 2014;10(1):e1003900.

17. Ho CC, Liao WY, Wang CY, Lu YH, Huang HY, Chen HY, Chan WK, Chen HW, Yang PC. TREM-1 expression in tumor-associated macrophages and clinical outcome in lung cancer. Am J Respir Crit Care Med. 2008;177(7):763-70.

18. Duan M, Wang ZC, Wang XY, Shi JY, Yang LX, Ding ZB, Gao Q, Zhou J, Fan J. TREM-1, an inflammatory modulator, is expressed in hepatocellular carcinoma cells and significantly promotes tumor progression. Ann Surg Oncol. 2015;22(9):3121-9.

19. Kuemmel A, Alflen A, Schmidt LH, Sebastian M, Wiewrodt R, Schulze AB, Buhl R, Radsak M. Soluble Triggering Receptor Expressed on Myeloid Cells 1 in lung cancer. Sci Rep. 2018;8(1):10766.

20. Wu Q, Zhou W, Yin S, Zhou Y, Chen T, Qian J, Su R, Hong L, Lu H, Zhang F, et al. Blocking Triggering Receptor Expressed on Myeloid Cells-1-Positive Tumor-Associated Macrophages Induced by Hypoxia Reverses Immunosuppression and Anti-Programmed Cell Death Ligand 1 Resistance in Liver Cancer. Hepatology. 2019;70(1):198-214.

21. Verhaak RG, Hoadley KA, Purdom E, Wang V, Qi Y, Wilkerson MD, Miller CR, Ding L, Golub T, Mesirov JP, et al. Integrated genomic analysis identifies clinically relevant subtypes of glioblastoma characterized by abnormalities in PDGFRA, IDH1, EGFR, and NF1. Cancer Cell. 2010;17(1):98-110. 
22. Gill BJ, Pisapia DJ, Malone HR, Goldstein H, Lei L, Sonabend A, Yun J, Samanamud J, Sims JS, Banu $\mathrm{M}$, et al. MRI-localized biopsies reveal subtype-specific differences in molecular and cellular composition at the margins of glioblastoma. Proc Natl Acad Sci U S A. 2014;111(34):12550-5.

23. Iasonos A, Schrag D, Raj GV, Panageas KS. How to build and interpret a nomogram for cancer prognosis. J Clin Oncol. 2008;26(8):1364-70.

24. Eckel-Passow JE, Lachance DH, Molinaro AM, Walsh KM, Decker PA, Sicotte H, Pekmezci M, Rice T, Kosel ML, Smirnov IV, et al. Glioma Groups Based on 1p/19q, IDH, and TERT Promoter Mutations in Tumors. N Engl J Med. 2015;372(26):2499-508.

25. Brennan CW, Verhaak RG, McKenna A, Campos B, Noushmehr H, Salama SR, Zheng S, Chakravarty D, Sanborn JZ, Berman SH, et al. The somatic genomic landscape of glioblastoma. Cell. 2013;155(2):462-77.

26. Huang da W, Sherman BT, Lempicki RA. Systematic and integrative analysis of large gene lists using DAVID bioinformatics resources. Nat Protoc. 2009;4(1):44-57.

27. Quail DF, Joyce JA. The Microenvironmental Landscape of Brain Tumors. Cancer Cell. 2017;31(3):326-41.

28. Yoshihara K, Shahmoradgoli M, Martinez E, Vegesna R, Kim H, Torres-Garcia W, Trevino V, Shen H, Laird PW, Levine DA, et al. Inferring tumour purity and stromal and immune cell admixture from expression data. Nat Commun. 2013;4:2612.

29. Aran D, Hu Z, Butte AJ. xCell: digitally portraying the tissue cellular heterogeneity landscape. Genome Biol. 2017;18(1):220.

30. Roe K, Gibot S, Verma S. Triggering receptor expressed on myeloid cells-1 (TREM-1): a new player in antiviral immunity? Front Microbiol. 2014;5:627.

31. Rody A, Holtrich U, Pusztai L, Liedtke C, Gaetje R, Ruckhaeberle E, Solbach C, Hanker L, Ahr A, Metzler $D$, et al. T-cell metagene predicts a favorable prognosis in estrogen receptor-negative and HER2positive breast cancers. Breast Cancer Res. 2009;11(2):R15.

32. Zhang C, Zhang Z, Zhang G, Zhang Z, Luo Y, Wang F, Wang S, Che Y, Zeng Q, Sun N, et al. Clinical significance and inflammatory landscapes of a novel recurrence-associated immune signature in early-stage lung adenocarcinoma. Cancer Lett. 2020;479:31-41.

33. Marin-Acevedo JA, Dholaria B, Soyano AE, Knutson KL, Chumsri S, Lou Y. Next generation of immune checkpoint therapy in cancer: new developments and challenges. J Hematol Oncol. 2018;11(1):39.

34. Huang J, Liu F, Liu Z, Tang H, Wu H, Gong Q, Chen J. Immune Checkpoint in Glioblastoma: Promising and Challenging. Front Pharmacol. 2017;8:242.

35. Zhang X, Zhu S, Li T, Liu YJ, Chen W, Chen J. Targeting immune checkpoints in malignant glioma. Oncotarget. 2017;8(4):7157-74.

36. Xu P, Zhang X, Liu Q, Xie Y, Shi X, Chen J, Li Y, Guo H, Sun R, Hong Y, et al. Microglial TREM-1 receptor mediates neuroinflammatory injury via interaction with SYK in experimental ischemic stroke. Cell Death Dis. 2019;10(8):555. 
37. Wu J, Li J, Salcedo R, Mivechi NF, Trinchieri G, Horuzsko A. The proinflammatory myeloid cell receptor TREM-1 controls Kupffer cell activation and development of hepatocellular carcinoma. Cancer Res. 2012;72(16):3977-86.

38. Zhang C, Cheng W, Ren X, Wang Z, Liu X, Li G, Han S, Jiang T, Wu A. Tumor Purity as an Underlying Key Factor in Glioma. Clin Cancer Res. 2017;23(20):6279-91.

39. Pierobon D, Bosco MC, Blengio F, Raggi F, Eva A, Filippi M, Musso T, Novelli F, Cappello P, Varesio L, et al. Chronic hypoxia reprograms human immature dendritic cells by inducing a proinflammatory phenotype and TREM-1 expression. Eur J Immunol. 2013;43(4):949-66.

40. Schiechl G, Brunner SM, Kesselring R, Martin M, Ruemmele P, Mack M, Hirt SW, Schlitt HJ, Geissler EK, Fichtner-Feigl S. Inhibition of innate co-receptor TREM-1 signaling reduces CD4(+) T cell activation and prolongs cardiac allograft survival. Am J Transplant. 2013;13(5):1168-80.

41. Zangi L, Klionsky YZ, Yarimi L, Bachar-Lustig E, Eidelstein Y, Shezen E, Hagin D, Ito Y, Takai T, ReichZeliger $S$, et al. Deletion of cognate CD8 T cells by immature dendritic cells: a novel role for perforin, granzyme A, TREM-1, and TLR7. Blood. 2012;120(8):1647-57.

42. Lee JB, Ha SJ, Kim HR. Clinical Insights Into Novel Immune Checkpoint Inhibitors. Front Pharmacol. 2021;12:681320.

43. Reardon DA, Brandes AA, Omuro A, Mulholland P, Lim M, Wick A, Baehring J, Ahluwalia MS, Roth P, Bahr O, et al. Effect of Nivolumab vs Bevacizumab in Patients With Recurrent Glioblastoma: The CheckMate 143 Phase 3 Randomized Clinical Trial. JAMA Oncol. 2020;6(7):1003-10.

44. Schalper KA, Rodriguez-Ruiz ME, Diez-Valle R, Lopez-Janeiro A, Porciuncula A, Idoate MA, Inoges S, de Andrea C, Lopez-Diaz de Cerio A, Tejada S, et al. Neoadjuvant nivolumab modifies the tumor immune microenvironment in resectable glioblastoma. Nat Med. 2019;25(3):470-6.

\section{Figures}


A

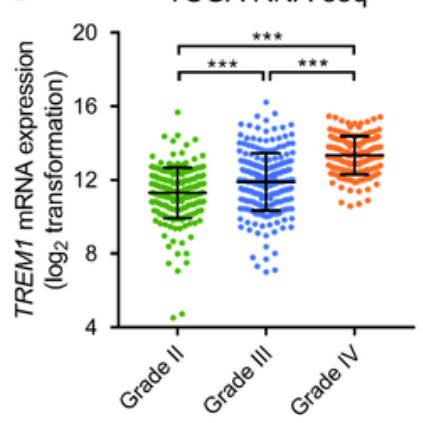

B

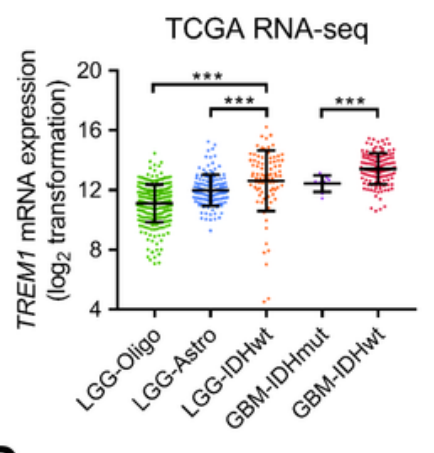

D

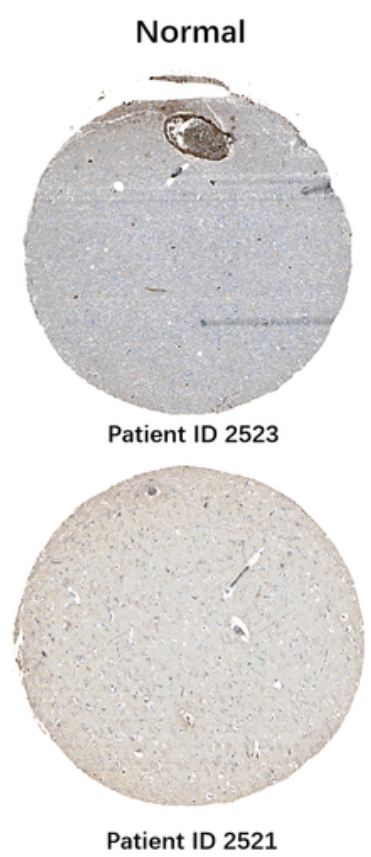

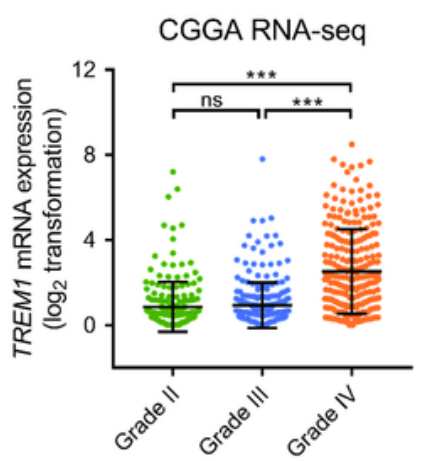

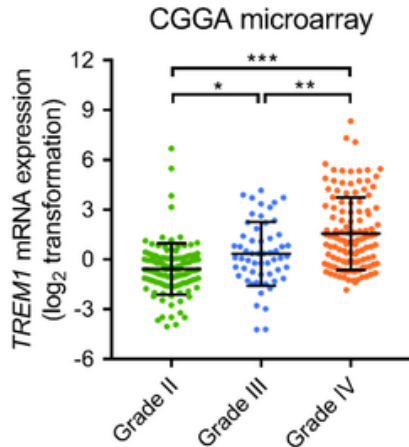

C

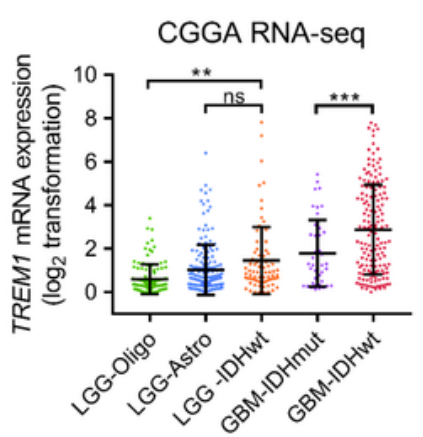

LGG

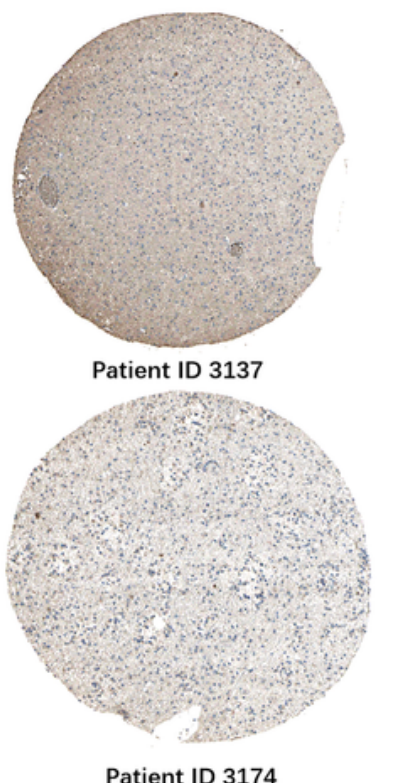

Patient ID 3174
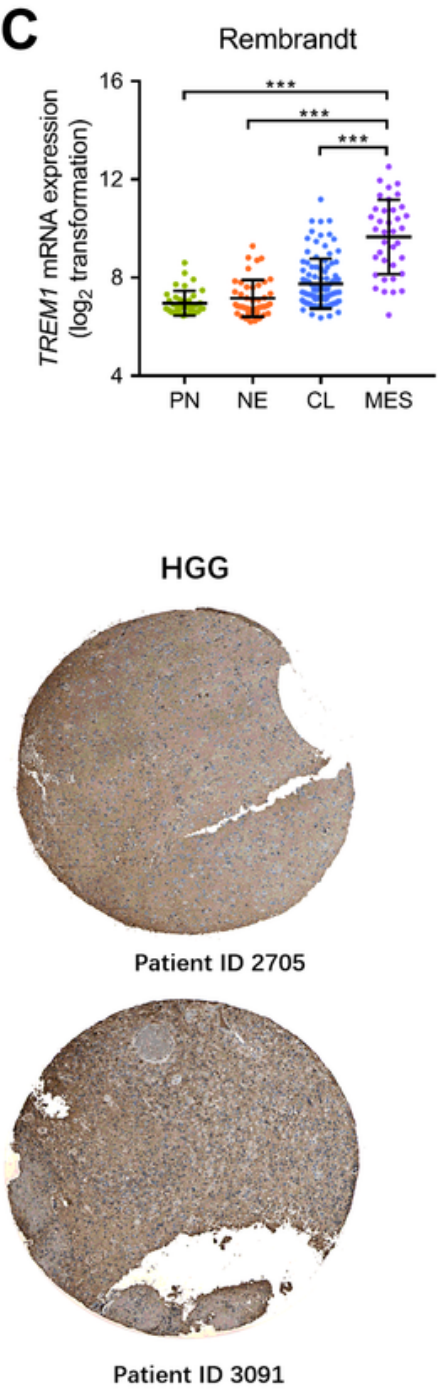

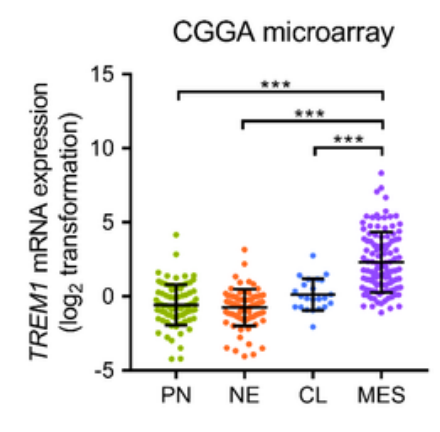

E
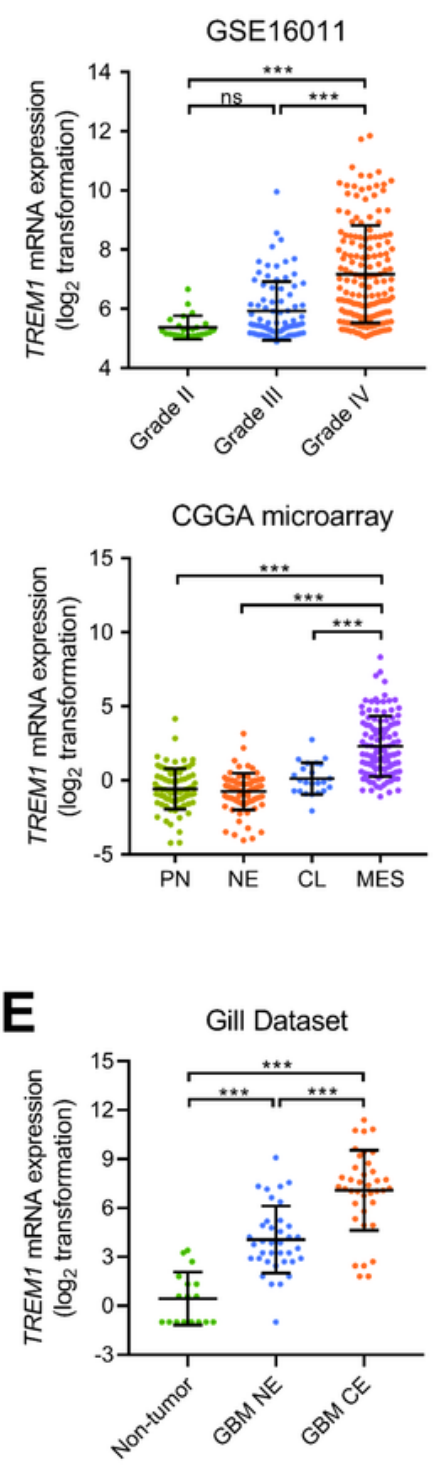

$\mathbf{F}$

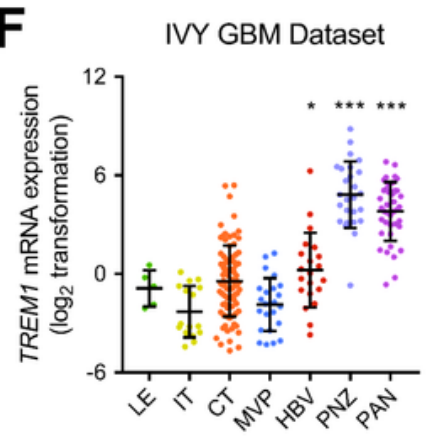

\section{Figure 1}

Landscape of molecular features associated with TREM1 expression. A. Analysis of TREM1 mRNA levels in WHO grade II-IV gliomas from TCGA, CGGA and GSE16011 datasets (TCGA RNA-seq, $n=669$; CGGA RNA-seq, $n=693$; CGGA microarray, $n=301$; GSE16011, $n=268$ ). B. TREM1 mRNA levels in gliomas from TCGA and CGGA datasets based on the 2016 WHO classification (TCGA RNA-seq, $n=669$; CGGA RNA-seq, $n=693$ ). C. Analysis of TREM1 protein levels in glioma tissues of different grades from the 
Human Protein Atlas. D. Analysis of TREM1 mRNA levels in the TCGA molecular subtype from the Rembrandt and CGGA datasets (Rembrandt, $n=580 ;$ CGGA microarray, $n=301$ ). The following abbreviations were used: PN (proneural), NE (neural), CL (classical), and MES (mesenchymal). E. Analysis of TREM1 mRNA levels in different radiographical regions of GBM in the Gill dataset $(n=92)$. $F$. Intratumor analysis of TREM1 expression using the IVY GBM database $(n=270)$. The following abbreviations were used: LE (Leading Edge), IT (Infiltrating Tumor), CT (Cellular Tumor), PAN (Pseudopalisading Cells Around Necrosis), PNZ (Peri-necrotic Zone), MVP (Microvascular Proliferation), and HBV (Hyperplastic Blood Vessels). Data are shown as the mean \pm SD for each group. ns, no significant difference, ${ }^{*} \mathrm{P}<0.05,{ }^{*} \mathrm{P}<0.01, * \star * \mathrm{P}<0.001$
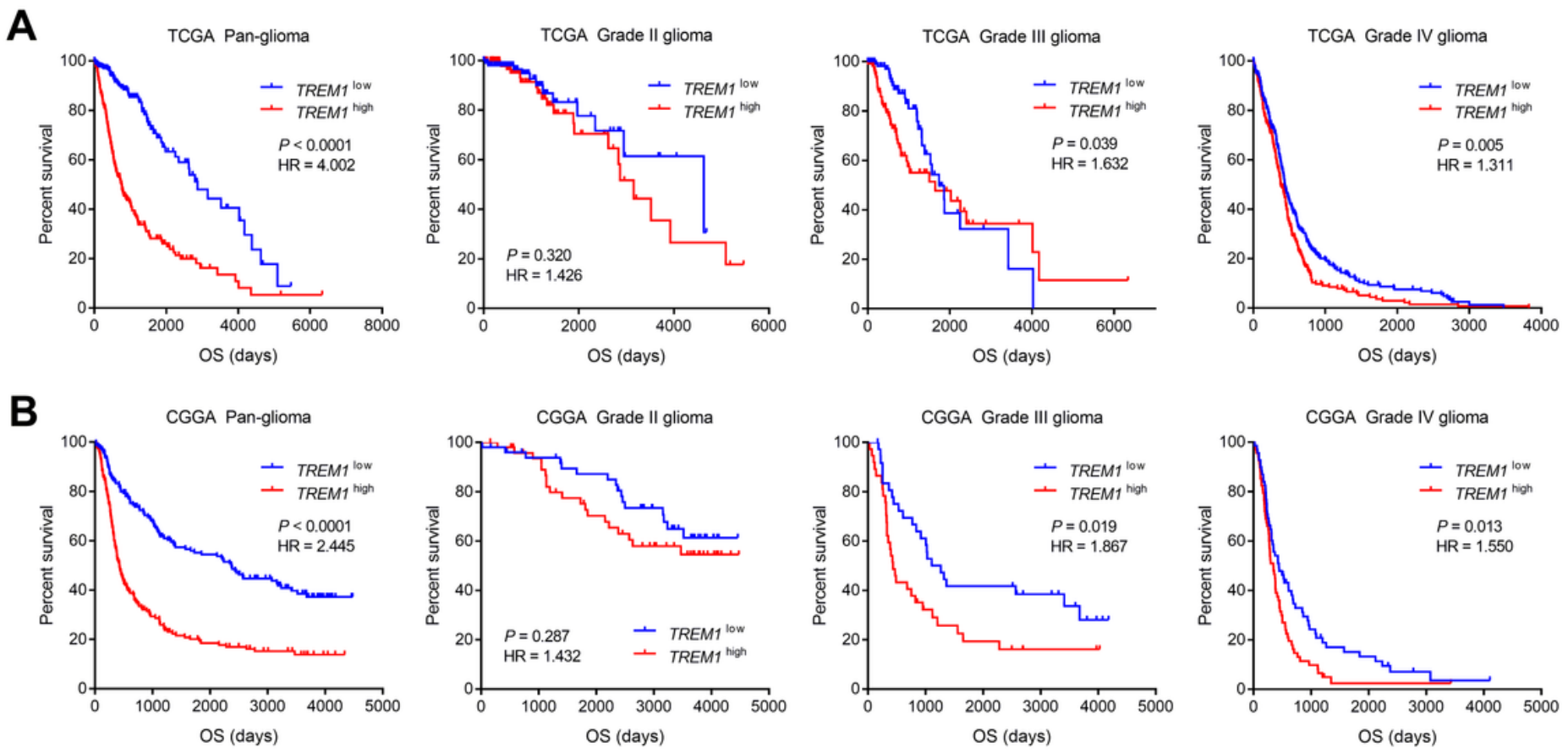

C
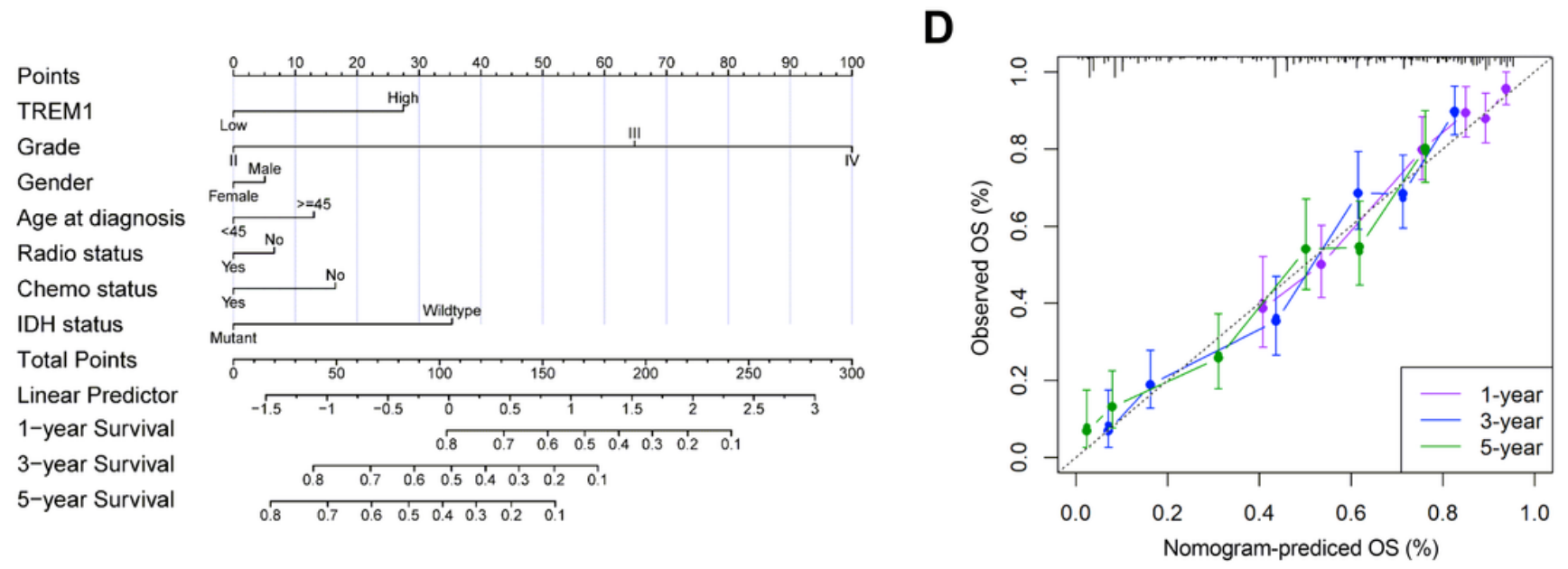

\section{Figure 2}

Clinical prognosis of patients with glioma with low and high TREM1 expression. A. and B. Kaplan-Meier analysis of overall survival (OS) based on high vs. low TREM1 expression in pan-glioma, grade II, grade III and grade IV GBM patients in TCGA and CGGA datasets. The median value of TREM1 expression was 
used as the cutoff value. C. Nomogram for predicting the survival probability of glioma patients. D. Calibration curves for predicting the 1-year, 3-year, and 5-year survival probability of glioma patients.
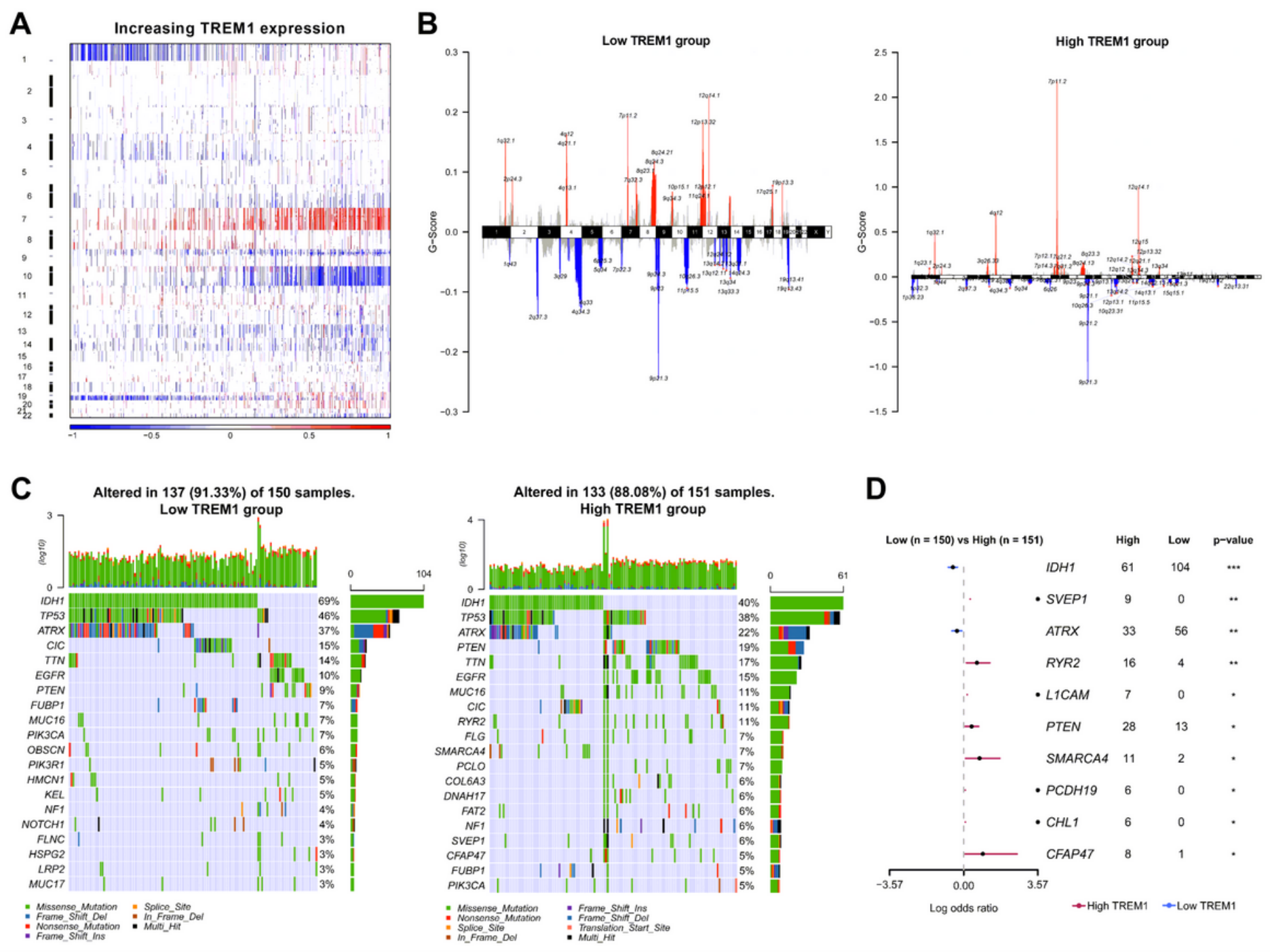

D

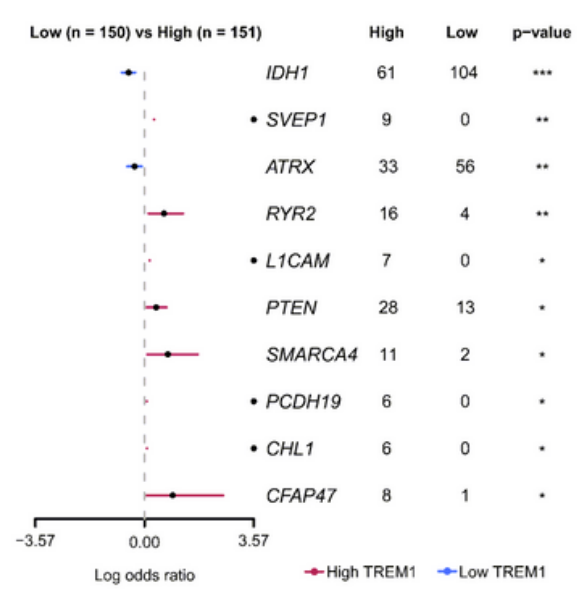

\section{Figure 3}

Distinct genomic changes associated with TREM1 expression. A. Overview of the copy number variation (CNV) profile in order of TREM1 expression. Blue (deletion); Red (amplification). B. Differential somatic mutations between gliomas with low and high TREM1 expression. C. Distinct CNV spectrum between gliomas with low and high TREM1 expression. D. Significant differentially mutated genes between gliomas with low and high TREM1 expression. 
A

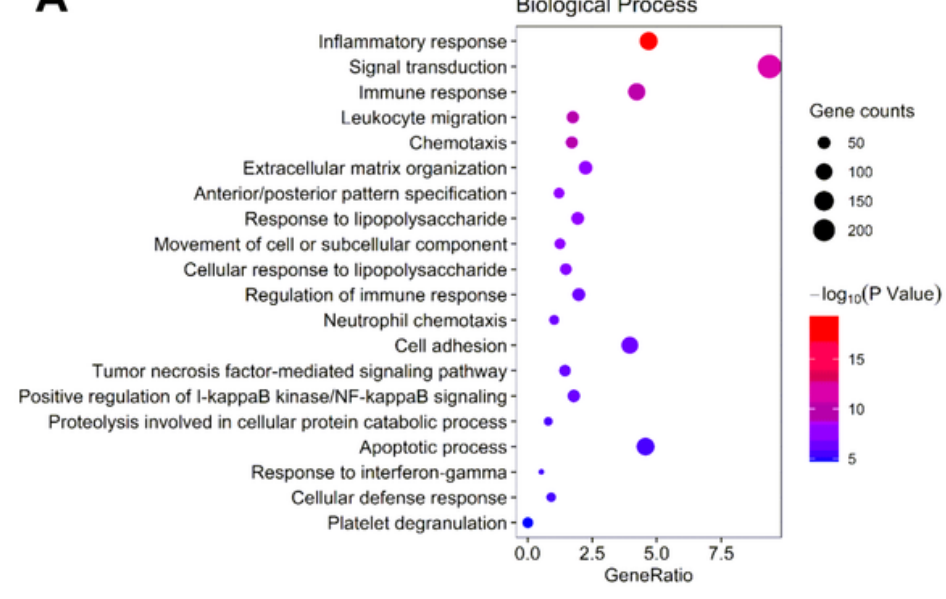

C TCGA RNA-seq
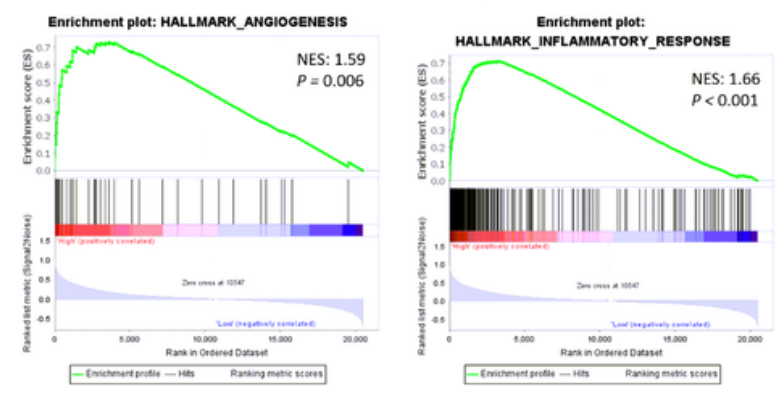

E

TCGA RNA-seq

HALLMARK_ANGIOGENESIS

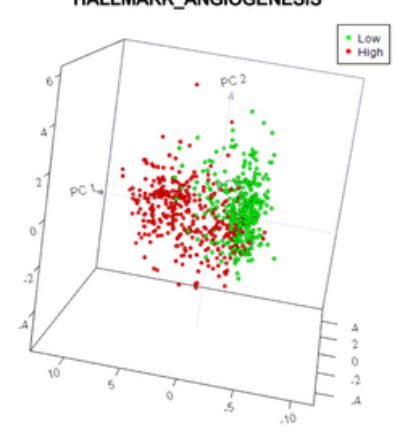

B

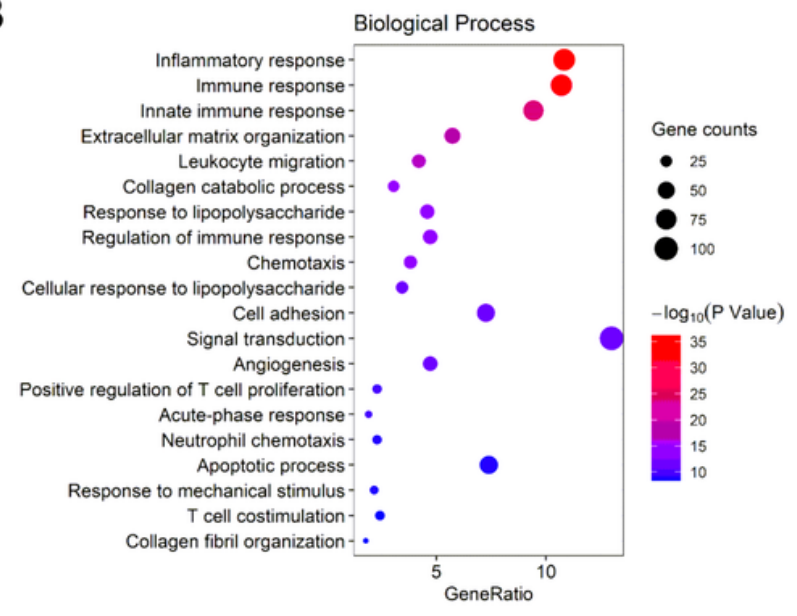

D

CGGA RNA-seq
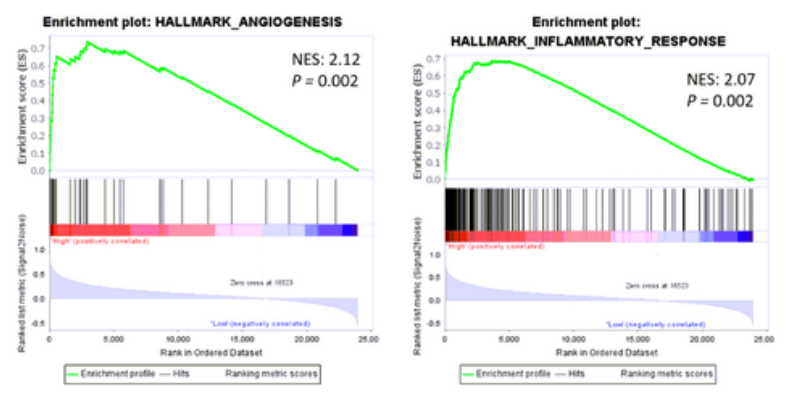

$\mathbf{F}$

CGGA RNA-seq
HALLMARK_ANGIOGENESIS

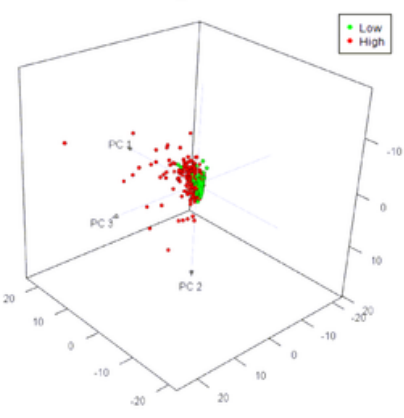

INFLAMMATORY_RESPONSE

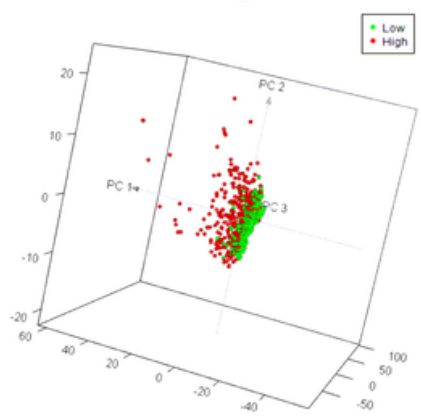

\section{Figure 4}

TREM1-related biological functions in glioma. A. and B. TREM1 associated with biological processes determined with GO analysis in the TCGA and CGGA datasets. C. and D. GSEA-validated biological processes related to TREM1 in TCGA and CGGA datasets. NES and P value were shown for each plot. E. and F. PCA analyzed biological processes (GO terms: ANGIOGENESIS and INFLAMMATORY RESPONSE) related to TREM1 expression levels in TCGA and CGGA datasets. 


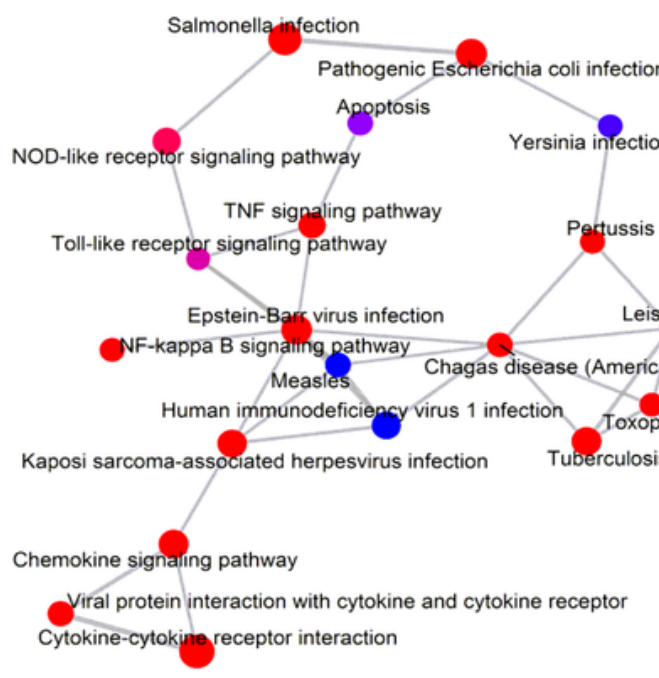

Fc gamma R-mediated phagocytosis

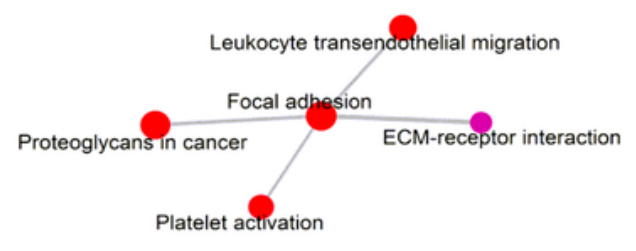

B

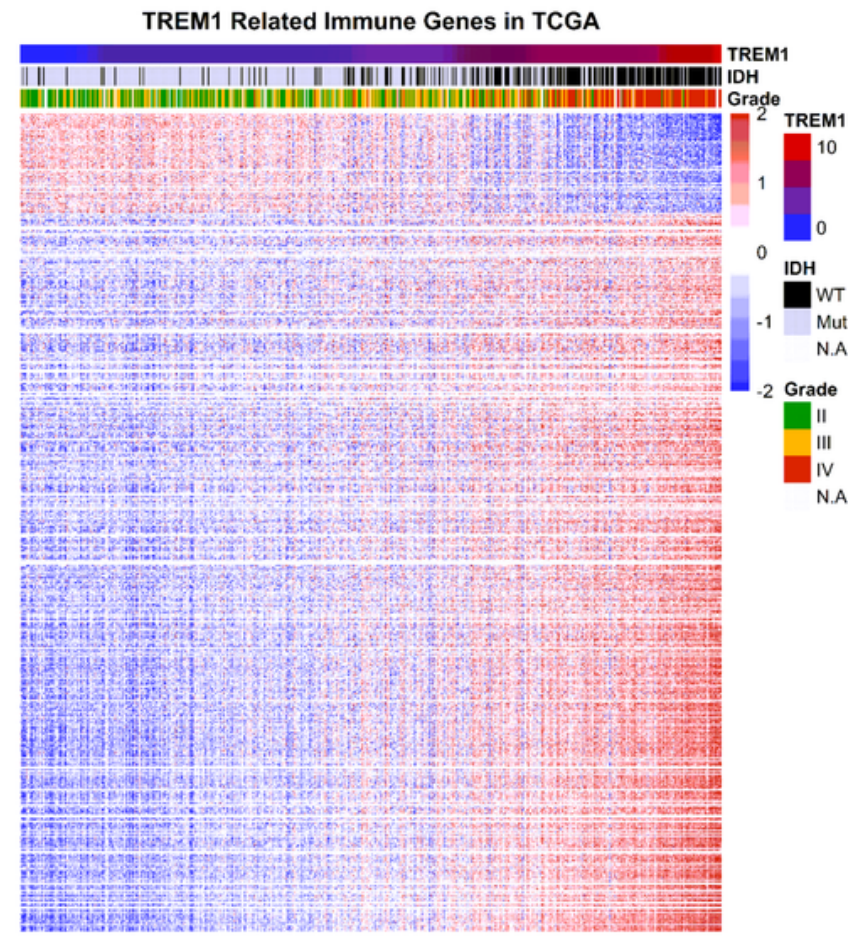

Hematopgietic cell lineage

Complement and coagulation cascades

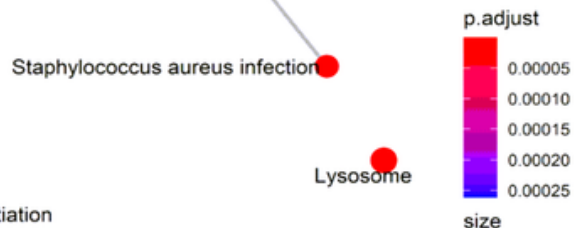

Rheumatoid arthritis?

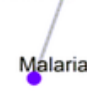

20

30

${ }_{50} 40$

8 Cytokine-cytokinte receptor interaction

C

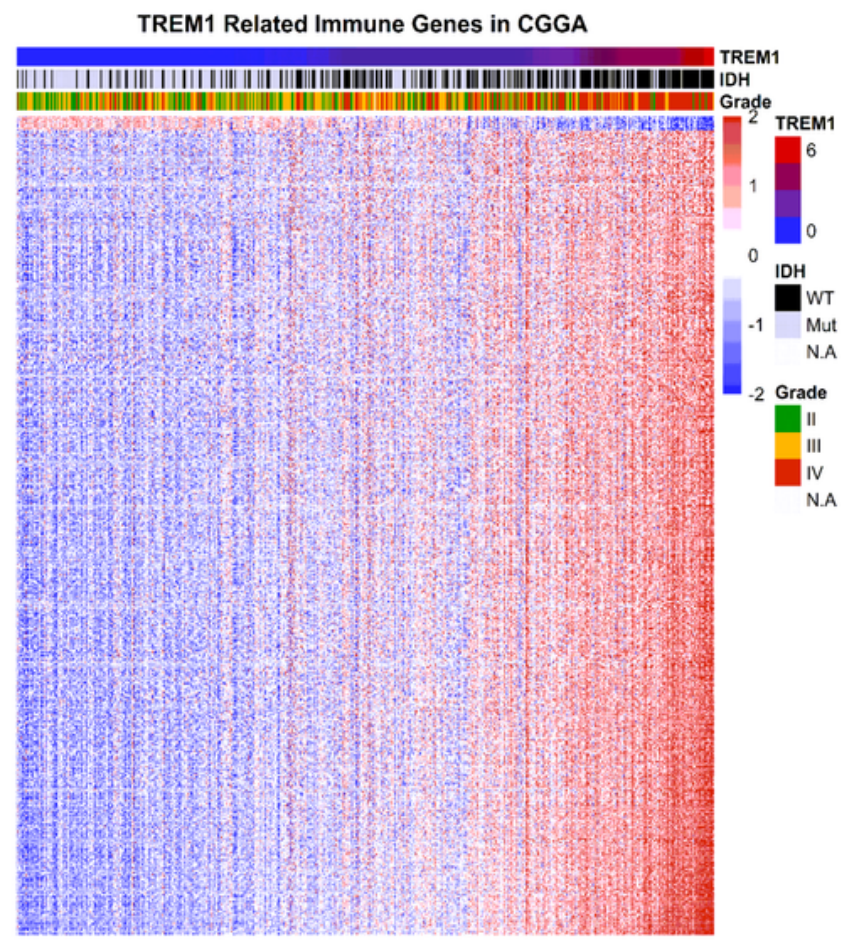

\section{Figure 5}

TREM1-related pathways and immune genes in glioma. A. Network construction of pathways enriched in TREM1-positively associated gene signatures in the TCGA cohort. B. Heatmap showing strong positive correlation of TREM1 with most immune genes. 
A TREM1 vs Tumor purity in TCGA-glioma patients

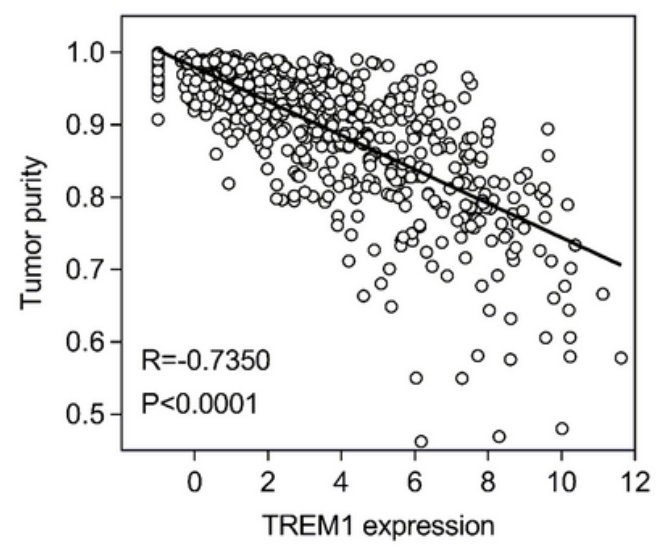

C

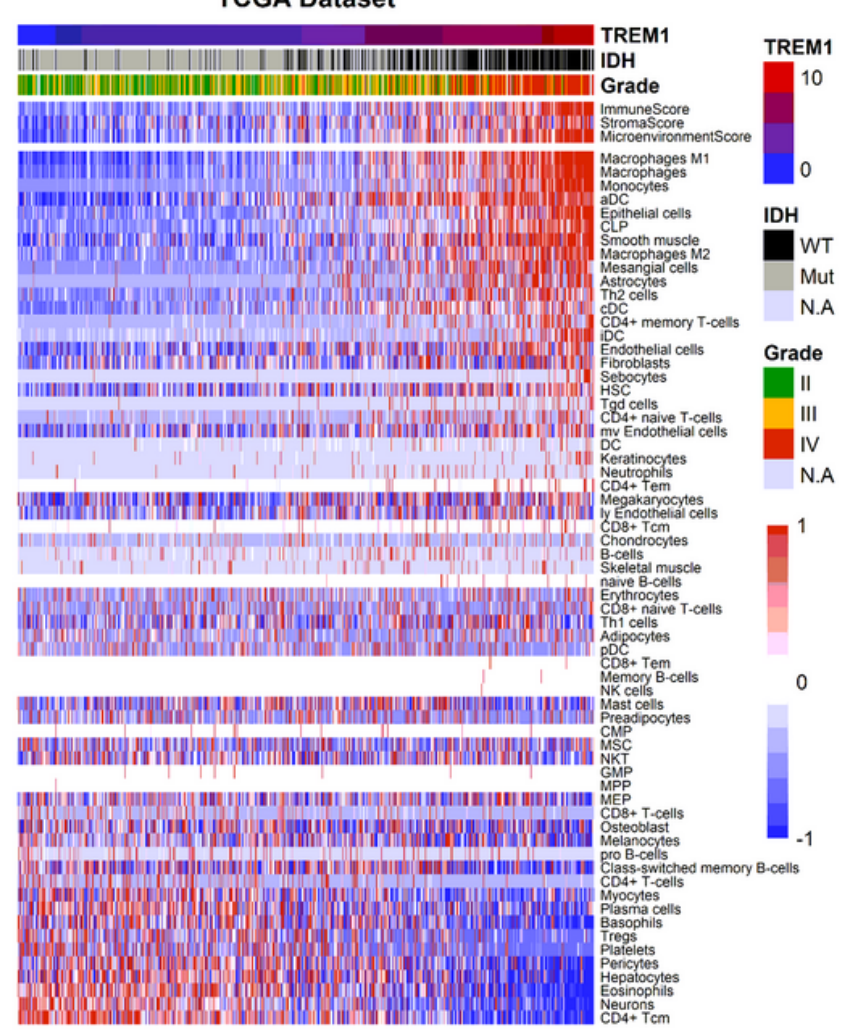

B TREM1 vs Tumor purity in CGGA-glioma patients

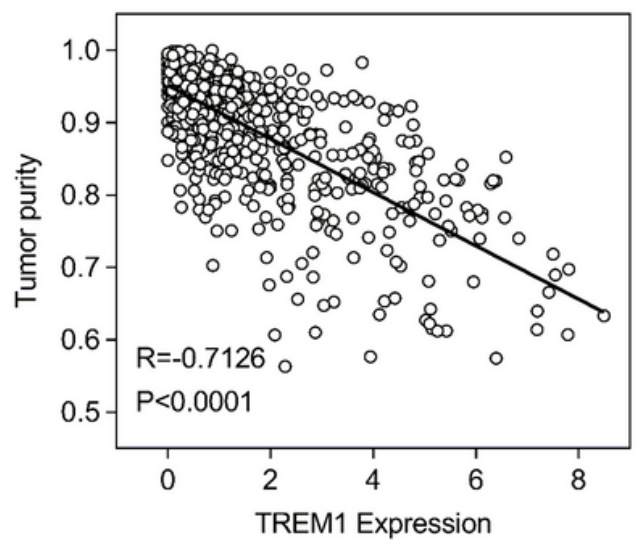

D

CGGA Dataset

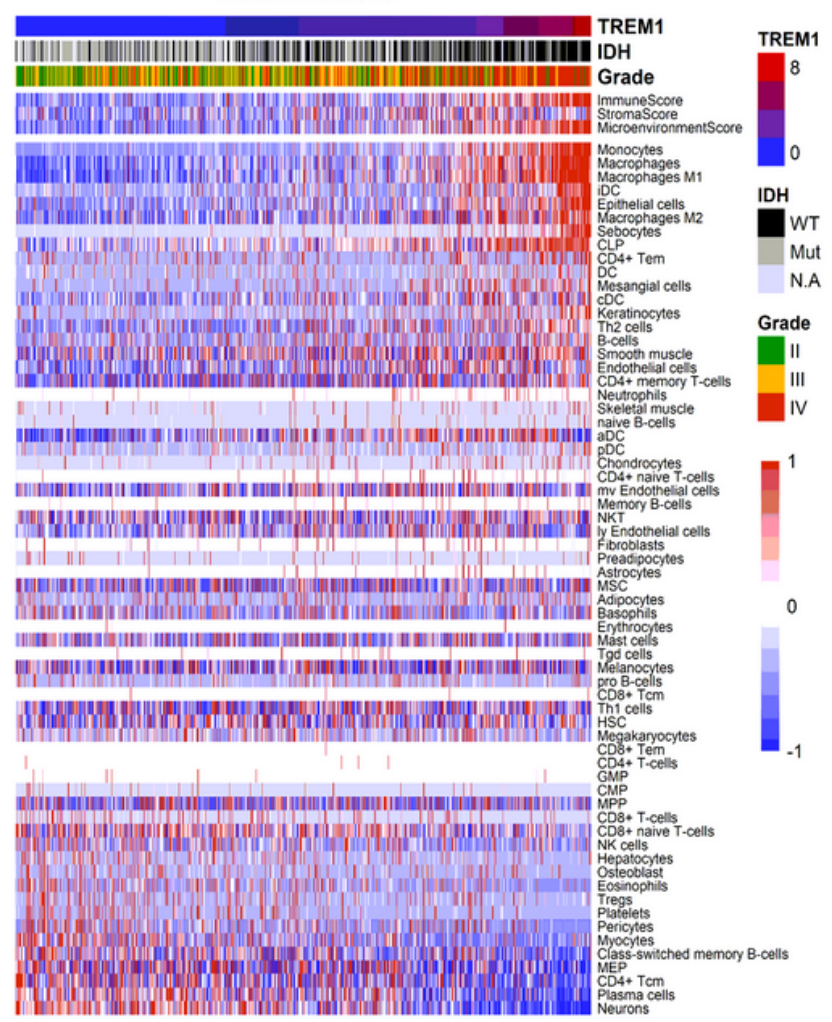

Figure 6

Association of TREM1 expression with tumor purity and immune and stromal cell populations in the glioma microenvironment. A. and B. Correlation analysis between TREM1 expression levels and tumor purity in TCGA and CGGA databases. C. and D. Heatmaps showing the relationship between TREM1 expression and immune and stromal cell populations in TCGA and CGGA datasets. Expression values were subjected to z-score normalization, with high levels shown in red and low levels shown in blue. 
A

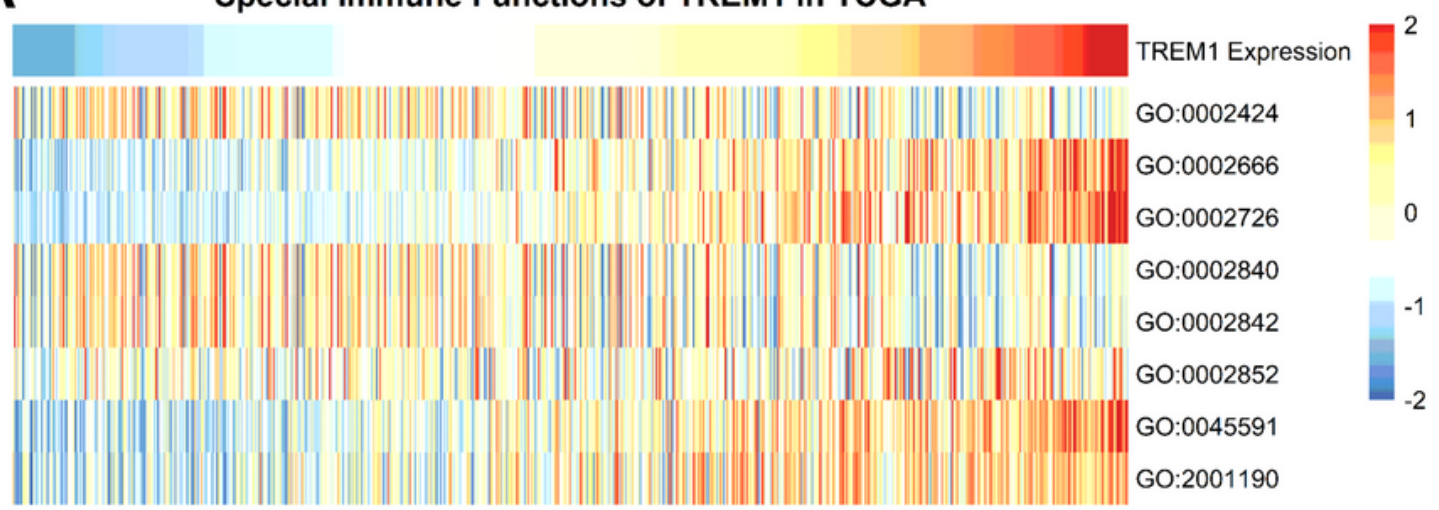

B

\section{Special Immune Functions of TREM1 in CGGA}

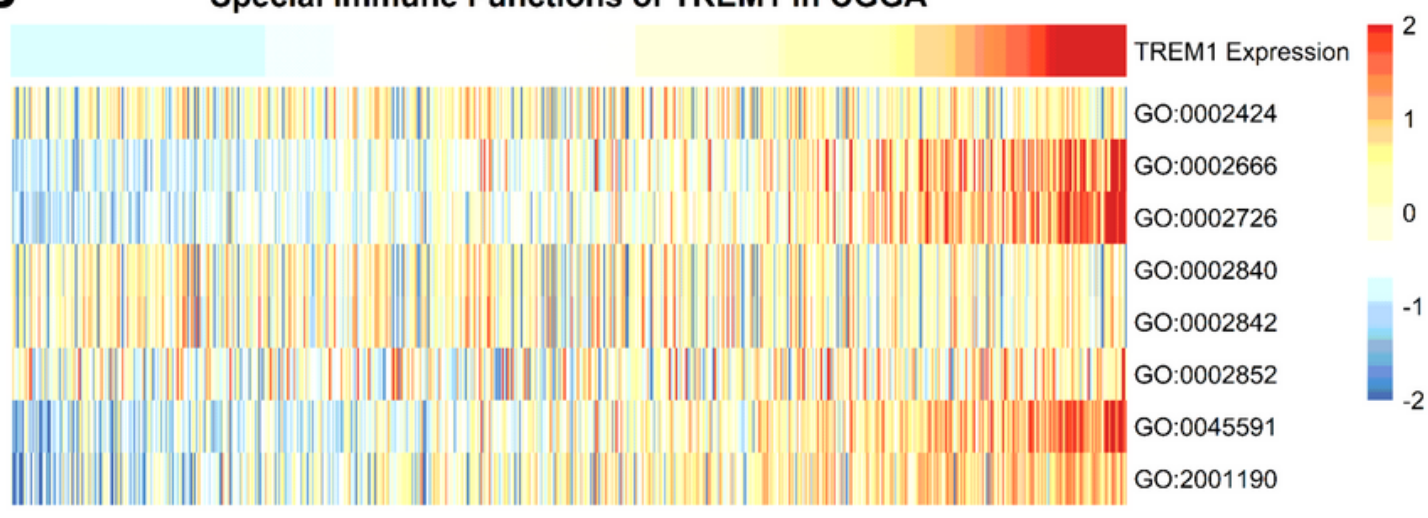

C

Correlogram of TREM1 and Inflammatory Activities TCGA Dataset

D CGGA Dataset
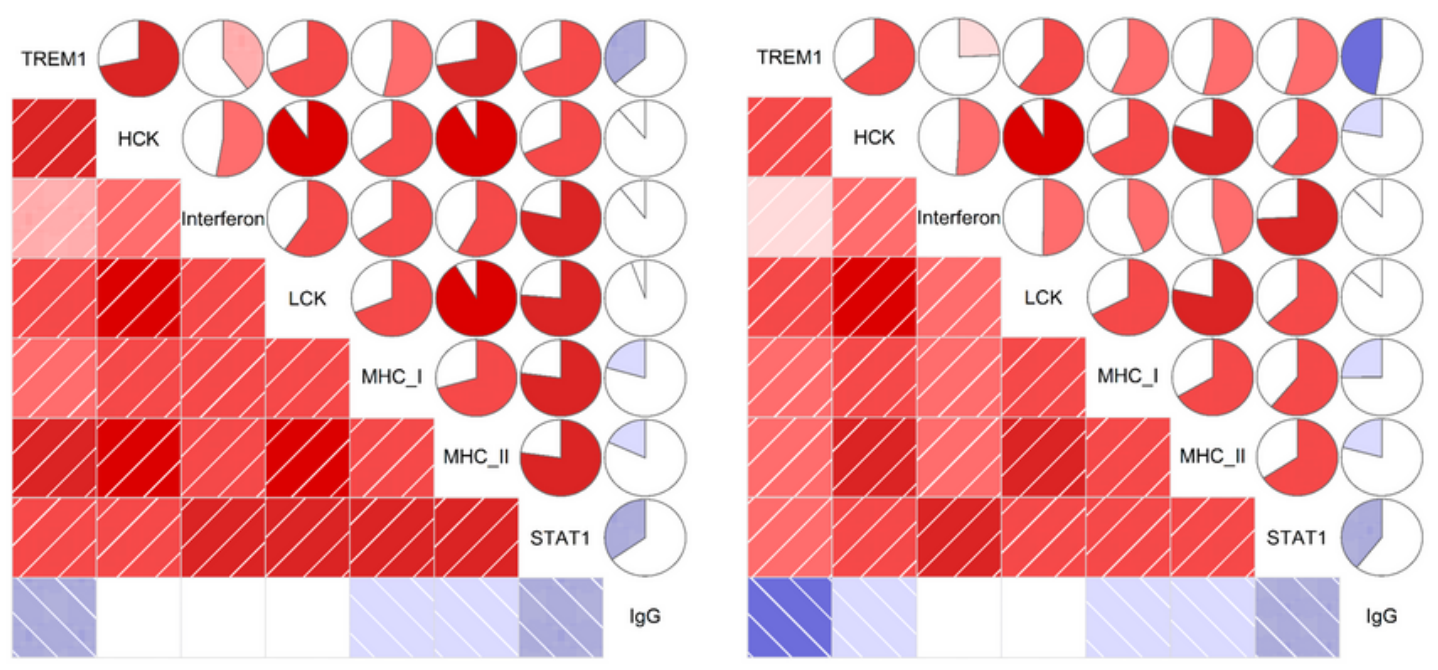

Figure 7

TREM1-related T cell immunity and inflammatory activities in glioma. A and B. Relationship between TREM expression level and T-cell-specific immunity. The following abbreviations were used: G0:0002424: T-cell-mediated immune response to tumor cell, G0:0002666: positive regulation of T-cell tolerance induction, G0:0002726: positive regulation of T-cell cytokine production, G0:0002840: regulation of T-cellmediated immune response to tumor cell, G0:0002842: positive regulation of T-cell-mediated immune 
response to tumor cell, G0:0002852: regulation of T-cell-mediated cytotoxicity directed against tumor cell target, GO:0045591: positive regulation of regulatory T-cell differentiation, GO:2001190: positive regulation of T-cell activation via T-cell receptor contact with antigen bound to $\mathrm{MHC}$ molecule on antigen presenting cell $\mathrm{C}$. and D. Correlogram of TREM1 and inflammatory metagenes in TCGA and CGGA cohorts.

A

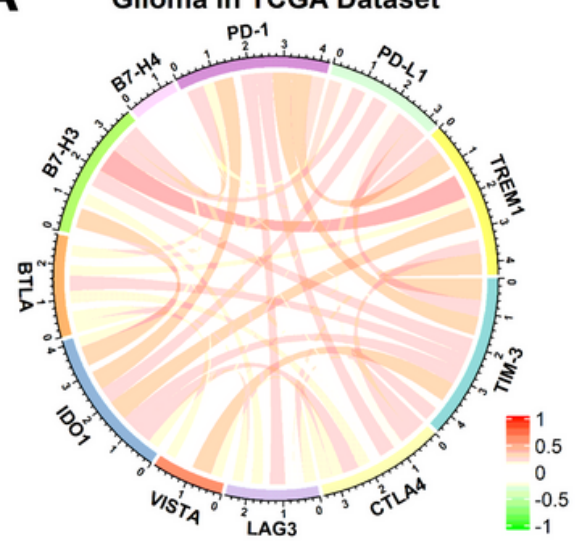

D

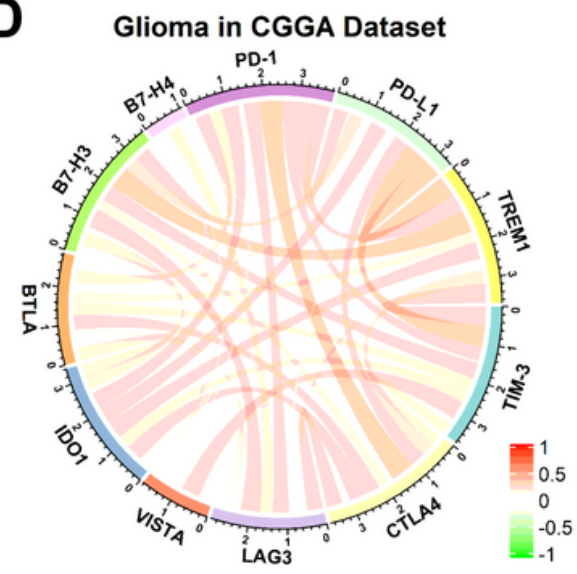

B

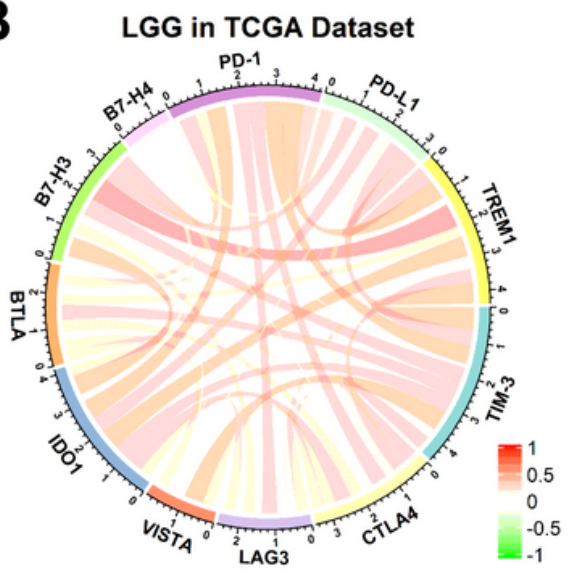

E

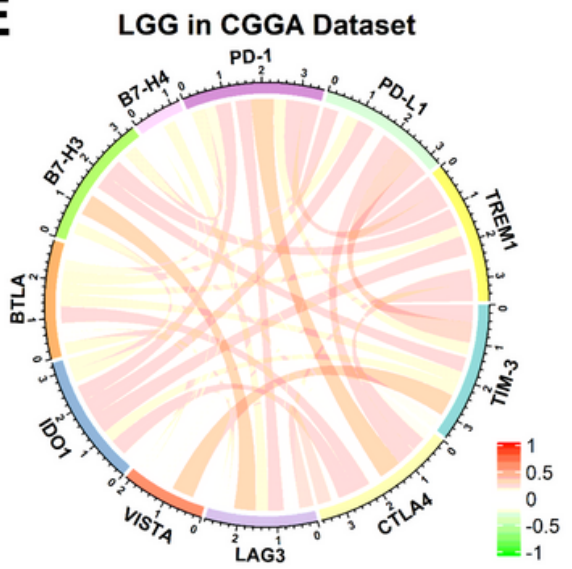

C GBM in TCGA Dataset

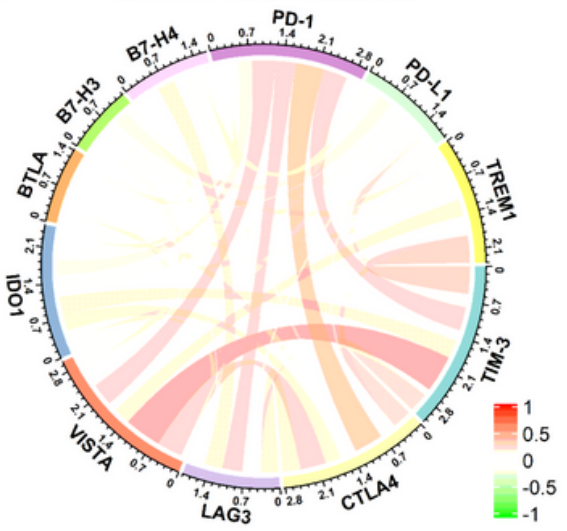

$\mathbf{F}$

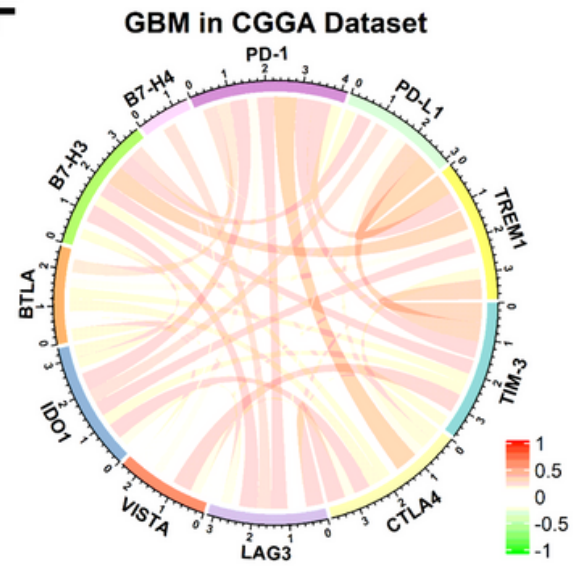

Figure 8

Correlation between TREM1 and other immune checkpoint members in pan-glioma (A, D), LGG (B, E), and GBM (C, F) from the TCGA and CGGA databases

\section{Supplementary Files}

This is a list of supplementary files associated with this preprint. Click to download.

- S1.tif

- S2.tif

- S3AUC.tif

- S4BPinGBM.tif 
- S5KEEGpathway.tif

- S6CGGAPPI.tif

- S7.tif

- S8.tif

- SupplementaryDatasetS1AmpDel.xlsx

- SupplementaryDatasetS2correlationgenes.xlsx

- SupplementaryDatasetS3immunegenes.xlsx

- SupplementaryDatasetS4infiltratingimmunecell.xlsx

- SupplementaryDatasetS5GOGSVA.xIsx

- SupplementaryDatasetS6ImmunecheckpointsinHeatmap.xlsx

- TableS1S2.docx 